\title{
GENERIC PROPERTIES OF POLYNOMIAL VECTOR FIELDS AT INFINITY
}

\author{
BY \\ ENRIQUE A. GONZÁLEZ VELASCO( $\left.{ }^{1}\right)$
}

\section{Introduction.}

1.1 In 1881 Poincare began the study of polynomial vector fields in the plane, $E^{2}$, by means of central projection of the paths on a sphere, $S^{2}$, tangent to the plane at the origin [1]. Thus, he provided the means for studying the behavior of the field in a neighborhood of infinity, which is represented by the equator, $S^{1}$.

This small part of Poincaré's Mémoire has later on been included in several texts [2], [3], [4], but always in a form similar to the original one. In $\$ 2$ we begin by clarifying it and writing it in modern terminology. More precisely, we consider $S^{2}$ as a differentiable manifold and obtain a field on $S^{2}$ which is canonically induced by the one in the plane, $X$, and which is analytic. We first obtain an induced field in the upper and lower hemispheres by central projection, and then we attempt to extend it to the equator, $S^{1}$. But this induced field blows up as we approach $S^{1}$ and the extension is not possible. However, if we multiply it by a certain factor which depends only on the degree of the polynomials in $X$, the extension is now possible. For the extended field, which we shall call the Poincare field and denote by $\pi(X), S^{1}$ is an invariant set, and the restriction of $\pi(X)$ to $S^{1}$ is a polynomial field multiplied by a nonvanishing analytic factor.

The underlying idea in Poincaré's work is to reduce the study of a field defined in the noncompact manifold $E^{2}$ to that of a field on the compact manifold $S^{2}$. This idea can be generalized to the $k$-dimensional case, and this forms the contents of the second part of $\$ 2$. Now the set of points at infinity is represented by the sphere $S^{k-1}$, which is also an invariant set. The restriction of the Poincaré field to $S^{k-1}$ will be denoted by $\pi_{\infty}(X)$ and, as before, it is a polynomial field multiplied by a nonvanishing analytic factor.

The factor which we use in the extension of the field to $S^{k-1}$ depends only on the degrees of the polynomials in $X$. Then we identify the set of all polynomial vector fields of a fixed degree with the Euclidean space of coefficients, $\mathscr{X}$, and our general goal is to look for properties of the field at infinity which are generic, that is, which

Received by the editors July 16, 1968 and, in revised form, October 30, 1968.

( $\left.{ }^{1}\right)$ This paper is derived from the author's doctoral thesis at Brown University, 1969. The author wishes to express his gratitude to his thesis advisor, Professor Mauricio M. Peixoto, for his support and unfailing aid at all times.

This research was supported in part by the National Science Foundation under Grant No. GP 7471. 
are true for a subset of $\mathscr{X}$ which is open and dense. So our work is along the lines of the "generic theory" of differential equations initiated by Peixoto [5], [6], Kupka [7], and Smale [8], [9]. But here the fact that the space $\mathscr{X}$ is so small and that every perturbation is global creates problems of a nature entirely different from that of those found in the usual generic theory.

In $\S 3$ we begin the study of the two dimensional case. If we denote by $\mathscr{G}$ the set of all fields in $\mathscr{X}$ for which $S^{1}$ contains only hyperbolic singularities of $\pi(X)$, or is a limit cycle with stability index different from zero, we prove that $\mathscr{G}$ is open and dense in $\mathscr{X}$. In $\S 4$ we prove that there is an open and dense subset, $\Sigma$, of $\mathscr{X}$ such that, for every $X \in \Sigma, \pi(X)$ is structurally stable in some neighborhood, $N$, of $S^{1}$ in $S^{2}$. The proof consists in showing that $\Sigma=\mathscr{G}$. It is interesting to note that we are able to know much more about the behavior at infinity than is known at a finite distance.

In $\S 5$ we turn our attention to the $k$-dimensional case. If we begin with $k=3$, it is natural to investigate whether or not the set of all $X \in \mathscr{X}$ for which $\pi_{\infty}(X)$ is structurally stable is open and dense. Since every perturbation in $\mathscr{X}$ is global, the natural idea to break any possible connection between saddle points is by a rotation of $\pi_{\infty}(X)$ on $S^{2}$. However, we show that, in general, it is not possible to obtain a rotation of $\pi_{\infty}(X)$ by a perturbation of $X$ in $\mathscr{X}$.

For this reason we restrict ourselves to the study of gradient fields in $\mathscr{X}$. For every such field, $X$, we prove that the nonwandering set, $\Omega$, of $\pi_{\infty}(X)$ is composed of singular points only. Moreover, in the set of all gradient vector fields in $\mathscr{X}$ there is an open and dense subset for which $\Omega$ is the union of a finite number of singular points, all of them hyperbolic. In this case we say that $\pi_{\infty}(X)$ is gradient-like.

Next, in $\S 6$, we consider the simplest possible case for $k=3$, that is, $\dot{x}=A x$ with $A$ a constant matrix. Then, for the corresponding Poincaré fields on $S^{3}$, we give a decomposition of the space of coefficients into a finite number of algebraic sets, each corresponding to a different qualitative behavior of $\pi(X)$. Hopefully, such analysis can be done for any $k$.

To sum up, in addition to its intrinsic interest, the investigation begun in this paper may, since the space $\mathscr{X}$ is finite dimensional, throw some light on the theory of bifurcations.

\section{Inducing a vector field on the sphere.}

2.1 In $k$ dimensional Euclidean space, $E^{k}$, we shall consider differential equations of the form $\dot{x}_{i}(t)=P_{i}\left(x_{1}(t), \ldots, x_{k}(t)\right)$, that is, vector fields of the form $X(x)=\left(P_{1}(x)\right.$, $\left.\ldots, P_{k}(x)\right)$, where the $P_{i}$ are polynomial functions in the variables $x_{1}$ to $x_{k}$. The set of all such vector fields for which the degree of any of the $P_{i}$ is $n$ or less will be denoted by $\mathscr{X}$. Our purpose is the study of the behavior of the paths at infinity for systems in $\mathscr{X}$.

$X$ is a field defined in $E^{k}$, a noncompact manifold. In this section, we shall find a field canonically induced by $X$ on the sphere $S^{k}$, a compact manifold. This will greatly simplify our work. The field on $S^{k}$ will be found to be analytic and its restriction to the equator, $S^{k-1}$, will behave essentially like a polynomial field. 
First, we shall present the case $k=2$, for which our intuitive insight is greater and the computational work reduced to a minimum. Then, we shall generalize our results for arbitrary $k$.

2.2 The method that we propose for the obtaining of the induced field is essentially due to Poincaré [1], and we shall present it here in modern terminology.

The idea is the following: we first consider $E^{2}$ imbedded in $E^{3}$ in such a way that, if $y=\left(y_{1}, y_{2}, y_{3}\right)$ represents an arbitrary point in $E^{3}$, then $E^{2}=\left\{y \in E^{3} \mid y_{3}=1\right\}$. Then we consider the sphere $S^{2}=\left\{y \in E^{3} \mid\|y\|=1\right\}$, which is tangent to $E^{2}$ at the north pole $(0,0,1)$. Finally, we shall consider the projection of the paths in $E^{2}$ into $S^{2}$ by means of central projection, and find the field on $S^{2}$ corresponding to the projected paths. In this way, the points at infinity in $E^{2}$ - two for every direction -are now in a one-to-one correspondence with the points in $S^{1}=\left\{y \in S^{2} \mid y_{3}=0\right\}$, as will be seen more clearly below. Our problem is thus reduced to the study of the field induced on $S^{2}$ in the vicinity of $S^{1}$, which from now on will be called the equator of $S^{2}$.

There is an important reason for using central projection instead of stereographic projection from the south pole, which would seem to be the most natural possibility, which is that we will focus our attention on the set of points at infinity and we will want this set to be as big as possible. Clearly, the equator $S^{1}$ is bigger than the south pole.

2.3 Let us go now into the analytical details. The sphere $S^{2}$ will be considered as a differentiable manifold. There will be six coordinate neighborhoods given by $U_{i}=\left\{y \in S^{2} \mid y_{i}>0, i=1,2,3\right\}$ and $V_{i}=\left\{y \in S^{2} \mid y_{i}<0, i=1,2,3\right\}$. The corresponding coordinate maps $\phi_{i}: U_{i} \rightarrow E^{2}$ and $\psi_{i}: V_{i} \rightarrow E^{2}$ are defined by:

$$
\phi_{i}(y)=\left(y_{j}, y_{k}\right) / y_{i}, \quad \text { and } \quad \psi_{i}(y)=\left(y_{j}, y_{k}\right) / y_{i}, \quad i, j, k=1,2,3 ; j<k .
$$

We shall denote by $z=\left(z_{1}, z_{2}\right)$ the value of $\phi_{i}(y)$ or $\psi_{i}(y)$ for any $i$, so that $z$ represents different things according to the case under consideration.

We have an important reason for choosing these neighborhoods and maps instead of using stereographic projection. The ones chosen will give us simpler expressions for the components of the induced field.

Now, the central projection of $E^{2}$ into $S^{2}$ associates two points of $S^{2}$ to every $x \in E^{2}$. It is defined by the functions:

$$
\begin{aligned}
& y=f(x)=\left(x_{1}, x_{2}, 1\right) / \Delta(x), \\
& y=f^{\prime}(x)=-\left(x_{1}, x_{2}, 1\right) / \Delta(x),
\end{aligned}
$$

where $\Delta(x)=\left(x_{1}^{2}+x_{2}^{2}+1\right)^{1 / 2}$.

The induced field will be obtained as follows: first, to the vector $X(x)$ at $x \in E^{2}$ we associate two vectors, $d f_{x}(X(x))$ at $y=f(x)$ and $d f_{x}^{\prime}(X(x))$ at $y=f^{\prime}(x)$, where $d f_{x}$ and $d f_{x}^{\prime}$ are the differentials of $f$ and $f^{\prime}$ respectively at the point $x$. We might expect that the two fields $d f_{x}(X(x))$ and $d f_{x}^{\prime}(X(x))$ could be extended to the equator; 
but we shall see that this is not so, since they blow up as we approach $S^{1}$. However, we have:

THEOREM 1. The field induced on the complement of the equator, $S^{2}-S^{1}$, by means of the differentials of $f$ and $f^{\prime}$ can be extended analytically to the whole of the sphere after multiplication by the factor $y_{3}^{n-1}$, and in such a way that the equator is invariant.

Proof. We begin by computing the differential and finding the explicit expression for the induced field for the case in which $y \in U_{1}$. The other cases would be studied in a similar way. We have, using (1) and (2):

$$
z=\phi_{1}(y)=\left(y_{2}, y_{3}\right) / y_{1}=\left(x_{2}, 1\right) / x_{1} .
$$

The differential of $f$ is represented by the matrix $\left(d f_{x}\right)_{i j}=\partial z_{i} / \partial x_{j}$ and, consequently, if $y=f(x)$, that is, if $y$ is in the upper hemisphere:

$$
d f_{x}=\left[\begin{array}{cc}
-x_{2} / x_{1}^{2} & 1 / x_{1} \\
-1 / x_{1}^{2} & 0
\end{array}\right]
$$

Then

$$
d f_{x}(X(x))=\left(1 / x_{1}\right)^{2}\left[x_{1} P_{2}(x)-x_{2} P_{1}(x),-P_{1}(x)\right] .
$$

We can write this in terms of the corresponding point on the sphere, $y=f(x)$, and get, from (3):

$$
d f_{x}(X(x))=\left(\frac{y_{3}}{y_{1}}\right)^{2}\left[\frac{y_{1}}{y_{3}} P_{2}\left(\frac{y_{1}}{y_{3}}, \frac{y_{2}}{y_{3}}\right)-\frac{y_{2}}{y_{3}} P_{1}\left(\frac{y_{1}}{y_{3}}, \frac{y_{2}}{y_{3}}\right),-P_{1}\left(\frac{y_{1}}{y_{3}}, \frac{y_{2}}{y_{3}}\right)\right] .
$$

If $y \in f^{\prime}(x)$, i.e., if $y$ is the lower hemisphere, we would start by computing $d f_{x}^{\prime}$ instead of $d f_{x}$. It is easy to check that we arrive again at the same expression (6).

Therefore, we have induced a vector field on $U_{1}$ which is defined and differentiable at all points except at $S^{1}=\left\{y \in S^{2} \mid y_{3}=0\right\}$ when $n>1$. However, if we multiply (6) by the factor $y_{3}^{n-1}$ we obtain a new field which is now differentiable in the whole of $U_{1}$. The factor $y_{3}^{n-1}$ depends only on the degree $n$ of the original system $X$, and the trajectories and critical points are the same before and after the application of the factor (except for a change in the direction of the arrows in the lower hemisphere when $n$ is even).

For analytical purposes it is convenient to express the obtained field in terms of the variable $z$. From (1) and (3) we get $y_{1} / y_{3}=1 / z_{2}, y_{2} / y_{3}=z_{1} / z_{2}$ and $y_{3}^{2}=z_{2}^{2} / \Delta(z)^{2}$. Now, in $U_{1}$ we have $y_{1}>0$ and then from (1) we know that $y_{3}$ is of the same sign as $z_{2}$. Thus $y_{3}=z_{2} / \Delta(z)$. Therefore, if we multiply (6) by $y_{3}^{n-1}$ and change the notation as indicated, we arrive at the final expression for the field in $U_{1}$

$$
\frac{z_{2}^{n}}{\Delta(z)^{n-1}}\left[P_{2}\left(\frac{1}{z_{2}}, \frac{z_{1}}{z_{2}}\right)-z_{1} P_{1}\left(\frac{1}{z_{2}}, \frac{z_{1}}{z_{2}}\right),-z_{2} P_{1}\left(\frac{1}{z_{2}}, \frac{z_{1}}{z_{2}}\right)\right] \text {. }
$$


Straightforward modifications will take care of the cases $y \in U_{2}$ or $y \in U_{3}$. We get the expressions:

$$
\frac{z_{2}^{n}}{\Delta(z)^{n-1}}\left[P_{1}\left(\frac{z_{1}}{z_{2}}, \frac{1}{z_{2}}\right)-z_{1} P_{2}\left(\frac{z_{1}}{z_{2}}, \frac{1}{z_{2}}\right),-z_{2} P_{2}\left(\frac{1}{z_{2}}, \frac{z_{1}}{z_{2}}\right)\right]
$$

for $U_{2}$, and for $U_{3}$

$$
\frac{1}{\Delta(z)^{n-1}}\left[P_{1}\left(z_{1}, z_{2}\right), P_{2}\left(z_{1}, z_{2}\right)\right]
$$

For the case in which $y \in V_{i}, i=1,2,3$, we obtain the same expressions (7) to (9) except for the following fact: in $V_{1}$ we have $y_{1}<0$ and then $y_{3}$ and $z_{2}$ are of different sign. Thus $y_{3}=-z_{2} / \Delta(z)$ and (7) must be multiplied by $(-1)^{n-1}$ to obtain the field in $V_{1}$. Also, it can be seen that (8) and (9) have to be multiplied by $(-1)^{n-1}$. The field which we have just obtained will be referred to as the Poincaré field and will be denoted by $\pi(X)$.

An essential feature of this field is that the equator, $S^{1}$, is always an invariant set. This fact can be seen as follows: for any $y \in S^{1}$ we have $y_{3}=0$, and since $z_{2}=y_{3} / y_{i}, i=1,2$, we get $z_{2}=0$. Now, the tangent vector at $y \in S^{1}$ is given by (7) or a similar expression, and we see that $z_{2}=0$ implies that the second component of the tangent vector is always zero. Therefore $S^{1}$ is invariant. Moreover, the restriction of $\pi(X)$ to $S^{1}$ behaves essentially like a polynomial field except for the factor $1 /\left(z_{1}^{2}+1\right)^{(n-1) / 2}$, which is always positive. These two features will have fruitful consequences in the remaining sections.

2.4 We conclude this part with two remarks. First, we have restricted our attention to the set $\mathscr{X}$ of all polynomial vector fields of degree $n$. It should be noted that these are not the only fields for which the construction of the Poincaré field is possible. Actually, it can be obtained for all those fields whose rate of growth at infinity is not bigger than that of systems in $\mathscr{X}$.

In [10] Lefschetz suggests that Poincaré's method amounts to transforming the differential equation in the plane into another in the projective plane. This is correct if by differential equation we understand a line element field, but not necessarily so if it means a vector field. In this connection, we notice that the integral curves in $S^{2}$ are always symmetric about the origin, but the Poincaré field is not symmetric. For example, if $n$ is even and $x \in E^{2}$ is a contracting node, we obtain a contracting node in the upper hemisphere by means of central projection, but in the lower hemisphere the factor $y_{3}^{n-1}$ is negative, and we have an expanding node. This fact immediately suggests the kind of problems that we would find in the use of the projective plane. Actually, it can be shown that, even if we use two different factors, one for each hemisphere, no extension of the field is possible to the projective plane for even $n$.

2.5 The general case, when $k$ is arbitrary, is dealt with in exactly the same way. The compact manifold on which we get the induced field is now $S^{k}=\left\{y \in E^{k} \mid\|y\|\right.$ 
$=1\}$, the equator $S^{k-1}=\left\{y \in S^{k} \mid y_{k+1}=0\right\}$ and the $2(k+1)$ coordinate neighborhoods are $U_{i}=\left\{y \in S^{k} \mid y_{i}>0, i=1, \ldots, k+1\right\}$ and $V_{i}=\left\{y \in S^{k} \mid y_{i}<0, i=1, \ldots\right.$, $k+1\}$. The coordinate maps $\phi_{i}: U_{i} \rightarrow E^{k}$ and $\psi_{i}: V_{i} \rightarrow E^{k}$ are now defined by

$$
\phi_{i}(y)=\left(1 / y_{i}\right)\left(y_{1}, \ldots, \hat{y}_{i}, \ldots, y_{k+1}\right),
$$

where the symbol $\wedge$ indicates that the $i$ th element is deleted, and similarly for the $\psi_{i}$ 's. We now give the corresponding $k$-dimensional versions of expressions (2) to (7) and of Theorem 1:

$$
\begin{aligned}
& y=f(x)=(1 / \Delta(x))\left(x_{1}, \ldots, x_{k}, 1\right), \\
& y=f^{\prime}(x)=-(1 / \Delta(x))\left(x_{1}, \ldots, x_{k}, 1\right),
\end{aligned}
$$

with $\Delta(x)=\left(x_{1}^{2}+\cdots+x_{k}^{2}+1\right)^{1 / 2}$;

$$
z=\phi_{i}(y)=\left(1 / x_{i}\right)\left(x_{1}, \ldots, \hat{x}_{i}, \ldots, x_{k}, 1\right) .
$$

THEOREM 1*. The field induced on the complement of the equator, $S^{k}-S^{k-1}$, by means of the differentials of $f$ and $f^{\prime}$ can be extended analytically to the whole of the sphere after multiplication by the factor $y_{k+1}^{n-1}$, and in such a way that the equator is invariant.

(4)*

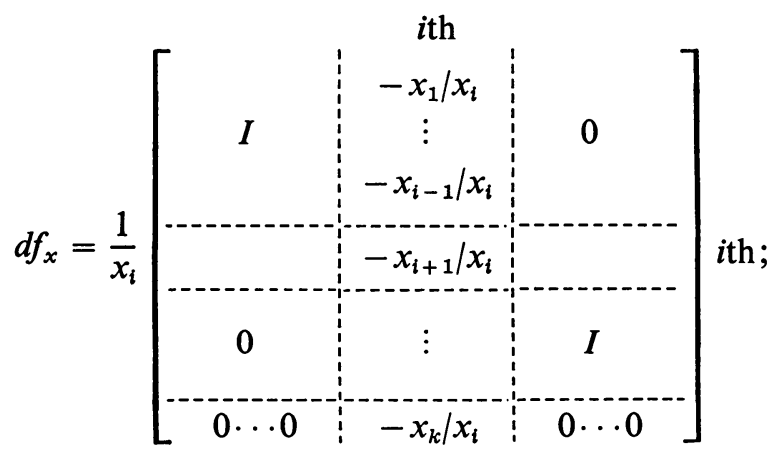

and then, for $j$ and $l$ such that $1 \leqq j<i, i \leqq l<k$,

$$
\begin{array}{r}
\begin{aligned}
d f_{x}(X(x))=\left(1 / x_{i}\right)^{2}\left[\ldots, x_{i} P_{j}(x)-x_{j} P_{i}(x), \ldots,\right. & x_{i} P_{l+1}(x) \\
& \left.-x_{l+1} P_{i}(x), \ldots,-P_{i}(x)\right] \\
d f_{x}(X(x))=\left(\frac{y_{k+1}}{y_{i}}\right)^{2}\left[\ldots, \frac{y_{i}}{y_{k+1}} P_{j}(\tilde{y})-\frac{y_{j}}{y_{k+1}} P_{i}(\tilde{y}), \ldots, \frac{y_{i}}{y_{k+1}} P_{1}(\tilde{y})\right. & \\
& \left.-\frac{y_{l+1}}{y_{k+1}} P_{i}(\tilde{y}), \ldots,-P_{i}(\tilde{y})\right],
\end{aligned}
\end{array}
$$

where $\tilde{y}=\left(1 / y_{k+1}\right)\left(y_{1}, \ldots, y_{k}\right)$; and

$$
\frac{z_{k}^{n}}{\Delta(z)^{n-1}}\left(\ldots, P_{j}(\tilde{z})-z_{j} P_{i}(\tilde{z}), \ldots, P_{l+1}(\tilde{z})-z_{l} P_{i}(\tilde{z}), \ldots,-z_{k} P_{i}(\tilde{z})\right)
$$

where

$$
\tilde{z}=\left(1 / z_{k}\right)\left(z_{1}, \ldots, 1, \ldots, z_{k-1}\right)
$$


As in the two-dimensional case, it is easy to see that the equator, $S^{k-1}$, is always an invariant set. The restriction of the Poincaré field to $S^{k-1}$ will be denoted by $\pi_{\infty}(X)$. As before, it should be noted that $\pi_{\infty}(X)$ behaves essentially like a polynomial field.

2.6 We shall illustrate our results with an example. For $k=3$ we consider the system

$$
\begin{aligned}
& P_{1}=-(x+y)\left(x^{2}+y^{2}-2 z^{2}\right), \\
& P_{2}=(x-y)\left(x^{2}+y^{2}-2 z^{2}\right), \\
& P_{3}=z\left(y^{2}-z^{2}\right) .
\end{aligned}
$$

To get the expression for $\pi_{\infty}(X)$ we use (7)* and put $z_{3}=0$. If we disregard the factor, we obtain the following expressions which are valid in $U_{1}, U_{2}$, and $U_{3}$ respectively:

$$
\begin{aligned}
& {\left[\left(1+s^{2}\right)\left(1+s^{2}-2 t^{2}\right), t\left(s^{2}-t^{2}\right)+t(1+s)\left(1+s^{2}-2 t^{2}\right)\right]} \\
& {\left[-\left(1+s^{2}\right)\left(1+s^{2}-2 t^{2}\right), t\left(1-t^{2}\right)+t(1-s)\left(1+s^{2}-2 t^{2}\right)\right]} \\
& {\left[(s+t)\left(2-s^{2}-t^{2}\right)+s\left(1-t^{2}\right),(t-s)\left(2-s^{2}-t^{2}\right)+t\left(1-t^{2}\right)\right] .}
\end{aligned}
$$

Since $n$ is odd, the same expressions are valid in $V_{1}, V_{2}$, and $V_{3}$.

From the first and the second we learn that the set $t=0$ is invariant and has no zeros. In fact, it represents a closed orbit with stability index greater than zero, as can be checked by direct computation. The only singularities are in $U_{3}$ and $V_{3}$, and are: $(0,0),(1,1),(1,-1),(-1,-1)$, and $(-1,1)$. After a local study of the neighborhood of each of these points, we learn that the first is an expanding focus, the second and fourth are contracting foci, and the third and fifth are saddle points. This field is represented graphically in Figure 1, as seen from the origin of $U_{3}$.

In the next sections we shall study the behavior of the Poincare field at infinity in some particular cases.

\section{Behavior of the induced field at infinity for the 2-dimensional case.}

3.1 In this section, the study of the induced field at infinity will be done in two parts. In the first one we shall investigate under what conditions there are singular points or closed orbits at infinity, and of what types. In the second part we shall look for properties of the behavior at infinity which are generic. In order to define this genericity we need to assign a metric to the space $\mathscr{X}$ of all polynomial vector fields of degree $n$. This is done as follows: for every $X \in \mathscr{X}$ the set of coefficients of $P_{1}$ and $P_{2}$ represents a point in $R^{(n+1)(n+2)}$. If we give this space Euclidean structure, we can identify $\mathscr{X}$ and $E^{(n+1)(n+2)}$, and thus $\mathscr{X}$ becomes a complete metric space. Then we have:

Definition 1. A generic property of systems $X \in \mathscr{X}$ is a property which is true for a subset of $\mathscr{X}$ which is open and dense.

We turn now to the first part of our study. 


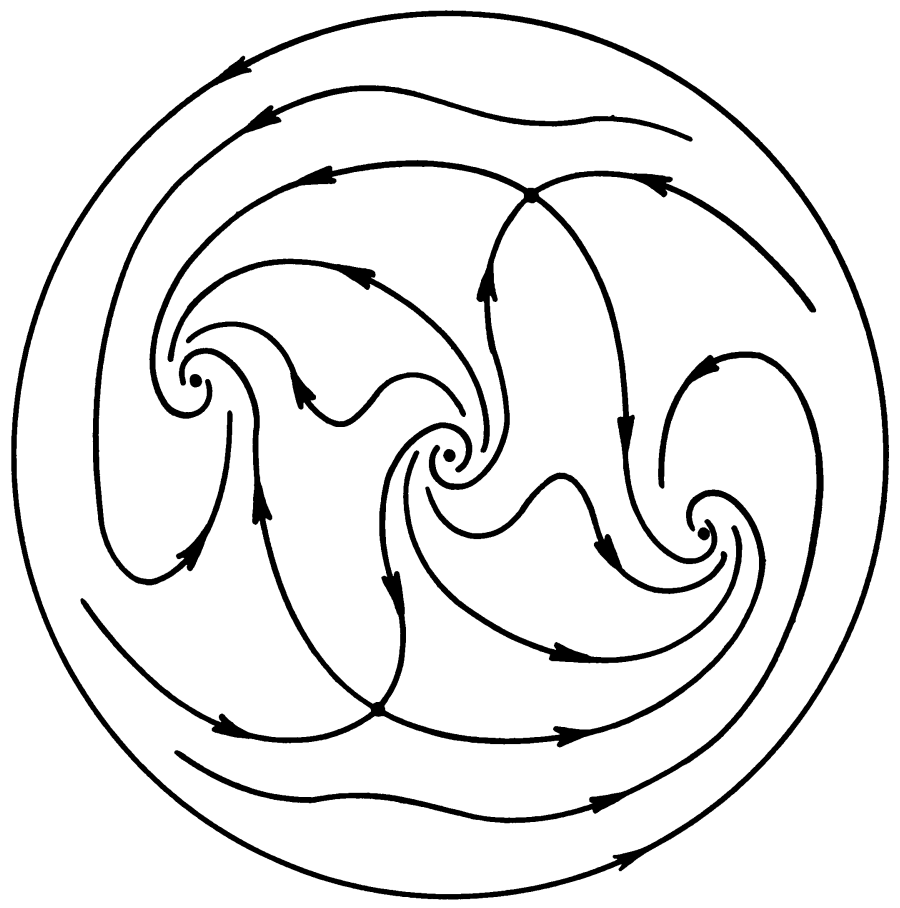

FIGURE 1

3.2 In the neighborhoods $U_{1}, U_{2}, V_{1}$, and $V_{2}$, which are the only ones containing points at infinity, our field is given by expressions (7) and (8). In the following discussion, we shall simplify the notation in order to avoid the use of the subscripts 1 and 2 , and shall write $X=(P, Q)$ instead of $X=\left(P_{1}, P_{2}\right)$ and $(s, t)$ instead of $\left(z_{1}, z_{2}\right)$.

With this convention in mind, we now recall that the equator, $S^{1}$, is an invariant set because the second component of (7) is zero for $t=y_{3} \Delta(z)=0$. Then, $\pi(X)$ will have a singular point in $S^{1} \cap\left(U_{1} \cup V_{1}\right)$ where the first component, $t^{n} Q(1 / t, s / t)$ $-s t^{n} P(1 / t, s / t)$, is also zero for $t=0$, or, in other words, where

$$
F(s)=Q_{n}(1, s)-s P_{n}(1, s)=0 .
$$

$P_{n}$ and $Q_{n}$ are the terms of degree $n$ of $P$ and $Q$. Likewise, from (8), we shall have a zero of the field in $S^{1} \cap\left(U_{2} \cup V_{2}\right)$ if

$$
G(s)=P_{n}(s, 1)-s Q_{n}(s, 1)=0 .
$$

Since we are working with polynomials, three different cases can occur: (A) $F(s) \equiv 0$, which implies $x Q_{n}(x, y)-y P_{n}(x, y) \equiv 0$ and then $G(s) \equiv 0 ;(\mathrm{B})$ Either $F(s)$ or $G(s)$ or both have isolated real zeros; (C) Neither $F(s)$ nor $G(s)$ have real zeros. 
In case (A), every point on the equator is a singular point; in case (B), the real zeros will give us singularities in $S^{1}$ which are isolated for $\pi_{\infty}(X)$; in case $(\mathrm{C})$, the equator is a closed orbit.

3.3 In the $(s, t)$ coordinates, let $(\alpha, 0)$ be one of the singularities from case (B). To analyze one such point we disregard the factor $1 / \Delta(z)^{n-1}$ in (7) and (8) and expand these expressions in a Taylor series about $(\alpha, 0)$. The linear part in either case is then given by the Jacobian matrix:

$$
\begin{aligned}
& J=\left[\begin{array}{cc}
\{d(F(s)) / d s\}_{s=\alpha} & Q_{n-1}(1, \alpha)-\alpha P_{n-1}(1, \alpha) \\
0 & -P_{n}(1, \alpha)
\end{array}\right], \\
& J=\left[\begin{array}{cc}
\{d(G(s)) / d s\}_{s=\alpha} & P_{n-1}(\alpha, 1)-\alpha Q_{n-1}(\alpha, 1) \\
0 & -Q_{n}(\alpha, 1)
\end{array}\right],
\end{aligned}
$$

if we are in either $U_{1}$ or $U_{2}$ respectively. For the $V$ 's multiply by $(-1)^{n-1}$. Since $J$ is a real triangular matrix its eigenvalues have nonzero real parts if and only if $\operatorname{det} J \neq 0$. If this is the case the singularities are nodes or saddle points according to whether the quotient of the eigenvalues is positive or negative. Summarizing, we have:

Proposition 1. Let $X \in \mathscr{X}$, then all the singularities of $\pi_{\infty}(X)$ in $S^{1}$ will be hyperbolic except in the following cases:

(a) $F(s)$ or $G(s)$ or both have multiple real zeros,

(b) $F(s)$ and $G(s)$ have simple real zeros, but $P_{n}(1, \alpha)=Q_{n}(1, \alpha)=0$ or $P_{n}(\alpha, 1)$ $=Q_{n}(\alpha, 1)=0$ for some real $\alpha$.

3.4 In case (C) the equator is a closed orbit. Then $n$ is odd. For suppose $n$ is even, then $x Q_{n}(x, y)-y P_{n}(x, y)$ is odd and will have a zero either for $x=0$ or for some finite value of $y / x$. In both cases we would have a singularity at infinity, contrary to the assumption.

Now, if $S^{1}$ is a limit cycle it will never be semistable. This is due to the fact that the field on $S^{2}$ is induced by means of central projection. The application of the factor $y_{3}^{n-1}$ with $n$ odd preserves the direction of the arrows in the lower hemisphere. Neither can $S^{1}$ be a composite limit cycle since, as our system is analytic, so is the Poincaré transformation.

Proposition 2. $S^{1}$ can be either a stable or unstable limit cycle or a closed orbit belonging to a ring of periodic orbits. Otherwise it contains singularities.

3.5 We begin now the second part of $\$ 3$.

We shall establish the following notation: the set of all systems $X \in \mathscr{X}$ having at least one nonhyperbolic singularity in $S^{1}$ will be denoted by $\mathscr{P}$, and the set of all $X \in \mathscr{X}$ such that $S^{1}$ either belongs to a ring of closed orbits or is a limit cycle with stability index equal to zero by $\mathcal{O}$. Then we write $\mathscr{G}=\mathscr{X}-(\mathscr{P} \cup \mathcal{O})$. Our goal is to prove that the fact that $X$ belongs to $\mathscr{G}$ is a generic property in the sense of Definition 1. We have:

THeOREM 2. $\mathscr{G}$ is open and dense in $\mathscr{X}$. 
Proof. First we show that $\mathscr{X}-\mathscr{P}$ is dense in $\mathscr{X}$. Let $X$ be arbitrary in $\mathscr{X}$. Then from Proposition 1 in 3.3 we know that case (a) is possible only if the discriminant $D(F)$ of $F(s)$ is zero (note that $D(F)=(D G)$ since both $F$ and $G$ come from $x Q-y P$ ) [11]. Case (b) is possible only if the resultant $R\left(P_{n}, Q_{n}\right)$ is zero (from the general expression for the resultant of two polynomials it is easy to see that $R\left(P_{n}(1, \alpha)\right.$, $\left.\left.Q_{n}(1, \alpha)\right)=R\left(P_{n}(\alpha, 1), Q_{n}(\alpha, 1)\right)\right)$ [11]. Thus, a system $X \in \mathscr{X}$ will be in $\mathscr{P}$ only if it belongs to the algebraic varieties $D(F)=0$ and/or $R\left(P_{n}, Q_{n}\right)=0$, none of which coincides with the whole space $\mathscr{X}$. This proves that $\mathscr{X}-\mathscr{P}$ is dense in $\mathscr{X}$.

We now prove that $\mathscr{X}-\mathcal{O}$ is dense in $\mathscr{X}$. Let $X$ be arbitrary in $\mathscr{X}$. If $S^{1}$ belongs to a ring of closed orbits of $\pi(X)$, then a small rotation of $X$ in the plane will turn $S^{1}$ into a limit cycle. After the rotation, $S^{1}$ is still a closed orbit because it is an invariant set. However, all the other closed orbits in the ring are broken. Otherwise, we would have a new ring of closed orbits transversal to the one before the rotation is applied, which is clearly impossible. Now, let $S^{1}$ be a limit cycle with stability index equal to zero, and let $s(\tau), t(\tau)$ be the parametric representation of the trajectories in a given coordinate neighborhood. Denote by $U_{i}(1)$ that portion of $S^{1} \cap U_{i}$ for which $|s| \leqq 1$, and similarly for the $V_{i}$. Then the stability index of $S^{1}$ is given by:

$$
h\left(S^{1}\right)=\sum_{i} \int_{U_{i}(1)} \operatorname{div} \pi(X) d \tau+\sum_{i} \int_{V_{i}(1)} \operatorname{div} \pi(X) d \tau .
$$

But $n$ is odd, as we have seen before, and we have $(-1)^{n-1}=1$. Then, in $V_{i}, \pi(X)$ is given by the same expression as in $U_{i}$ and we get:

$$
\begin{aligned}
h\left(S^{1}\right)= & 2 \sum_{i} \int_{U_{i}(1)} \operatorname{div} \pi(X) d \tau=2 \sum_{i} \int_{U_{i}(1)}\left[\frac{d \dot{s}}{d s}+\frac{d \dot{t}}{d t}\right]_{s=s(\tau) ; t=0} d \tau \\
= & 2 \int_{U_{1}(1)}\left\{\frac{d}{d s}\left[\frac{1}{\Delta^{n-1}}(F(s)+t \ldots)\right]\right. \\
& \left.-\frac{d}{d t}\left[\frac{1}{\Delta^{n-1}}\left(t P_{n}(1, s)+t^{2} \ldots\right)\right]\right\}_{s=s(\tau): t=0} d \tau \\
& +2 \int_{U_{2}(1)}\left\{\frac{d}{d s}\left[\frac{1}{\Delta^{n-1}}(G(s)+t \cdots)\right]\right. \\
= & 2 \int_{U_{1}(1)}\left[\frac{d}{d s} \frac{F(s)}{\Delta^{n-1}}-\frac{P_{n}(1, s)}{\Delta^{n-1}}\right]_{s=s(\tau): t=0} d \tau \\
& +2 \int_{U_{2}(1)}\left[\frac{d}{d s} \frac{G(s)}{\Delta^{n-1}}-\frac{Q_{n}(s, 1)}{\Delta^{n-1}}\right]_{s=s(\tau) ; t=0} d \tau .
\end{aligned}
$$

It is now clear that if this integral is zero for a given system $X=(P, Q)$ it will be different from zero for the perturbed system $\tilde{X}=\left(P+\varepsilon X^{n}, Q+\varepsilon x^{n-1} y\right)$, for which we obtain $-2 \varepsilon \int_{-1}^{1}\left[\left(1+s^{n-1}\right) / \Delta^{n-1}\right]_{t=0} d s$. This proves that $\mathscr{X}-\mathcal{O}$ is dense in $\mathscr{X}$. 
$\mathscr{X}-\mathscr{P}$ and $\mathscr{X}-\mathcal{O}$ are also open because, if $F(s)$ and $G(s)$ have simple real zeros which are not zeros of both $P_{n}$ and $Q_{n}$ at the same time, then this situation will be preserved under small perturbations of $X$. Also, if $h\left(S^{1}\right) \neq 0$, a small perturbation will not change this fact.

Therefore, $\mathscr{G}$ is the intersection of two open and dense sets and it is open and dense itself. This ends the proof of the theorem.

From the proof of the theorem we know that as long as we are away from the varieties $D(F)=0$ and $R\left(P_{n}, Q_{n}\right)=0$ in $\mathscr{X}$, we can be sure that the singularities in $S^{1}$, if any, are all hyperbolic. Also, if we consider those systems for which the integral (17) is not zero, $S^{1}$ cannot be a closed orbit of the type in $\mathcal{O}$. That is, given a field $X$ we have secured the means whereby to determine if it belongs to $\mathscr{G}$.

\section{Structural stability at infinity for the 2-dimensional case.}

4.1 Roughly speaking, a vector field defined on a compact manifold $M^{m}$ is said to be structurally stable if a "small" perturbation of $X$ does not change topologically the set of trajectories of $X$. This motivates the following definition:

Definition 2. $X \in \mathscr{X}$ is structurally stable at infinity if and only if there exists in $S^{2}$ a neighborhood, $N$, of the equator, $S^{1}$, and, for every $\varepsilon>0$, a neighborhood, $\mathscr{U}$, of $X$ in $\mathscr{X}$ such that, whenever $Y \in \mathscr{U}$, there exists an $\varepsilon$-homeomorphism, $h$, of $N$ onto $N$ transforming trajectories of $\pi(X)$ onto trajectories of $\pi(Y)$, and with $S^{1}$ an invariant set of $h$.

Our goal is twofold, to characterize the structurally stable systems, and to see how the set of all such systems, $\Sigma$, is imbedded in $\mathscr{X}$. This is done in the Theorem 3.

\subsection{TheOrem 3. $\Sigma=\mathscr{G}$, and therefore is open and dense in $\mathscr{X}$.}

Proof. We have only to prove that $\Sigma=\mathscr{G}$ and the rest is a consequence of Theorem 2.

We first show that $\Sigma \subset \mathscr{G}$. If $X \in \Sigma$, then from Theorem 2 we know that we can find a $Y \in \mathscr{U}$ such that $Y \in \mathscr{G}$. Then, the existence of the homeomorphism $h$ implies that $X$ is topologically conjugate to a system in $\mathscr{G}$. We have to show that it actually belongs to $\mathscr{G}$. Suppose, for instance, that $X \notin \mathscr{X}-\mathscr{P}$ and let $(\alpha, 0) \in U_{1}$ be a nonhyperbolic singularity of $\pi(X)$. Then, consider $\tilde{X}=\left(P+\varepsilon X^{n}, Q+\varepsilon \alpha\right)$. It can be seen, by using equations (12) and (14), that $\pi(\tilde{X})$ also has a singularity at $(\alpha, 0)$ which is hyperbolic, but the dimension of the stable manifold changes with the sign of $\varepsilon$. This contradicts the existence of the homeomorphism $h$, thus implying that $X$ has to be in $\mathscr{X}-\mathscr{P}$. Similarly, the perturbation given at the end of $\S 3$ shows that $X$ must be in $\mathscr{X}-\mathcal{O}$. Therefore, $X \in \mathscr{G}$, and so $\Sigma \subset \mathscr{G}$.

Now we show that $\mathscr{G} \subset \Sigma$, that is, that $X \in \mathscr{G}$ implies that $X \in \Sigma$. The proof will be done in two steps and will follow very closely those of Theorems 1 to 4 in [6]. First, we shall prove that if $X \in \mathscr{G}$ we can find a neighborhood, $N$, of $S^{1}$ in $S^{2}$ satisfying the following conditions:

C1. $N \cup \partial N$ contains no singularities or closed orbits other than those in $S^{1}$. 
C2. $\pi(X)$ is transversal to $\partial N$. This means that $\pi(X) \neq 0$ on $\partial N$, and that, in the tangent bundle of the sphere, denoted by $T\left(S^{2}\right)$, the submanifolds $\pi(X)\left(S^{2}\right)$ and $T(\partial N)$ intersect transversally.

C3. Each trajectory of $\pi(X)$ inside $N$ which meets $\partial N$ is tangent to it at most once.

C4. No trajectory of $\pi(X)$ connecting $\partial N$ to a saddle point in $N$ is tangent to $\partial N$.

For such a region $N$, we then prove that for all $Y \in \mathscr{X}$ close enough to $X$ we can construct the desired homeomorphism.

We begin by choosing a neighborhood, $N_{1}$, of $S^{1}$ so small that $\mathrm{Cl}$ is satisfied, but otherwise arbitrary. From standard transversality arguments it follows that there is a neighborhood, $N_{2}$, with $\partial N_{2}$ close to $\partial N_{1}$ and such that $\mathrm{C} 2$ is satisfied. The manifold of contacts, $\Gamma$, is a 0 -dimensional submanifold of $\partial N_{2}$.

Suppose now that $E$ is a point of $\partial N_{2}$ at which $\pi(X)$ is tangent from the inside, and let $\gamma$ be the trajectory through $E$. Then, if $\gamma$ goes to a saddle point or has more than one contact with $\partial N_{2}$, we proceed as follows: let $D$ be a small neighborhood of $E$ in $S^{2}$ such that, except $\gamma$, no other trajectory of $\pi(X)$ which is either tangent to $\ni N_{2}$ or goes to a saddle point meets $D$. Let $N_{3}$ be a new neighborhood such that $\partial N_{3}$ coincides with $\partial N_{2}$ outside $D$ and is very close to it in the inside, but with the contact at $E$ removed. In the tangent bundle, the new manifold of contacts, $\Gamma^{\prime}$, is still a 0 -dimensional submanifold of $\partial N_{3}$ because the transversality is preserved after the small perturbation, and $\Gamma^{\prime}$ is diffeomorphic to $\Gamma$. All the contacts are the same as before except for the one at $E$, which disappears, and which is replaced by a new contact in $D$. Because of the way in which $D$ was chosen, this new contact is such that the trajectory through it satisfies $\mathrm{C} 3$ and $\mathrm{C} 4$. We can repeat this procedure as many times as is necessary to get a neighborhood, $N$, for which all the conditions are satisfied.

Now we have to construct the $\varepsilon$-homeomorphism. The construction in [6] applies here mutatis mutandi. The differences are the following:

(i) Regarding Lemma 1 it is necessary to add that the singularity corresponding to the perturbed system is in $S^{1}$. Also, the new cycle of Lemma 2 is again $S^{1}$. This follows from the fact that $\mathscr{G}$ is open.

(ii) In Lemma 6 one of the $\alpha$ 's or $\omega$ 's may now be a saddle point instead of being a source or a sink. The lemma is still valid because $S^{1}$ is invariant for every $X \in \mathscr{G}$.

(iii) Canonical regions of types I, IV, and V are not possible now. The possible types are II, III, and III' (see Figure 2).

The construction of the $\varepsilon$-homeomorphism for this type is analogous to that for the type $\mathrm{V}$, with straightforward modifications. The mapping $\phi$ will now be $\phi: R^{*} \rightarrow U$ where $U$ is the rectangle $0 \leqq u \leqq 1,0 \leqq v \leqq 3$. This completes the proof of Theorem 3.

It is seen from the proof that we can get a definition of structural stability equivalent to Definition 2 if we suppress the requirement that the distance between $p \in N$ and $h(p) \in N$ must be less than $\varepsilon$. 


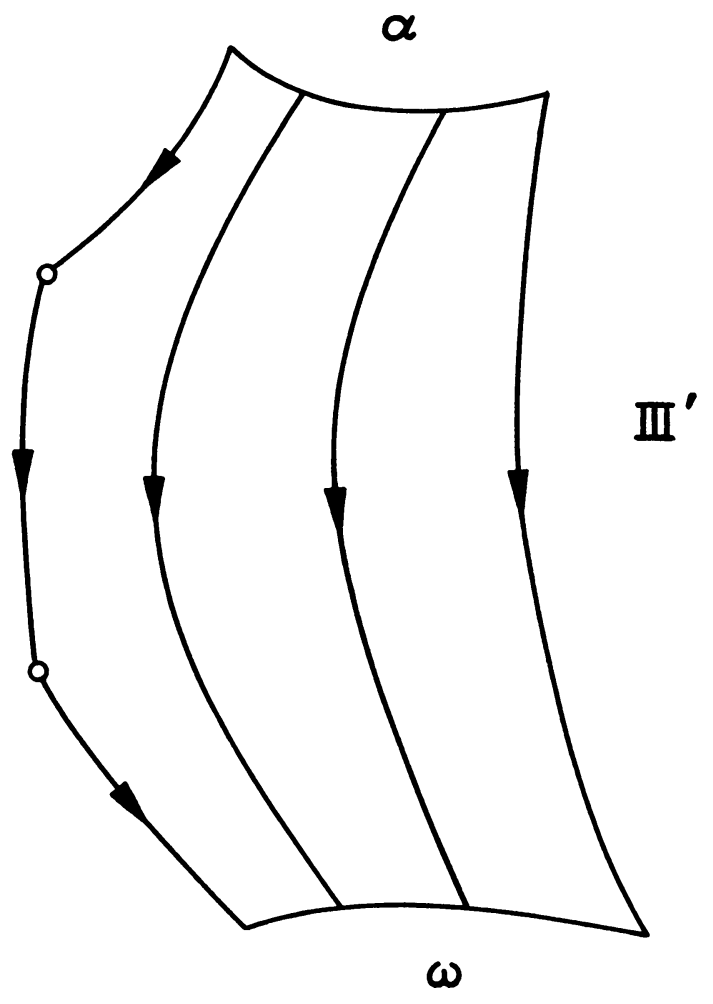

FigURE 2

\section{Behavior of the induced field at infinity for arbitrary $k$.}

5.1 Here we shall look for generic properties of the field $\pi_{\infty}(X)$. Thus, our goal is more restrictive than in $\S 3$, when we studied the behavior of $\pi(X)$ in a neighborhood of $S^{1}$.

In what follows we shall need the explicit expression for $\pi_{\infty}(X)$ (remember that $S^{k-1}$ is an invariant set). This is obtained from (7)* after putting $z_{k}=0$. For example, for $k=3$, if we write $(s, t)$ instead of $\left(z_{1}, z_{2}\right)$ and $P, Q, R$ instead of $P_{1}, P_{2}$, $P_{3}$, in order to simplify the notation, we obtain the following expressions for $\pi_{\infty}(X)$ which are valid in $U_{1}, U_{2}, U_{3}$ respectively:

$$
\begin{aligned}
& \frac{1}{\Delta(s, t)^{n-1}}\left(Q_{n}(1, s, t)-s P_{n}(1, s, t), R_{n}(1, s, t)-t P_{n}(1, s, t)\right), \\
& \frac{1}{\Delta(s, t)^{n-1}}\left(P_{n}(s, 1, t)-s Q_{n}(s, 1, t), R_{n}(s, 1, t)-t Q_{n}(s, 1, t)\right), \\
& \frac{1}{\Delta(s, t)^{n-1}}\left(P_{n}(s, t, 1)-s R_{n}(s, t, 1), Q_{n}(s, t, 1)-t R_{n}(s, t, 1)\right) .
\end{aligned}
$$

Here $P_{n}, Q_{n}$, and $R_{n}$ are the terms of degree $n$ of $P, Q$, and $R$ respectively. To obtain $\pi_{\infty}(X)$ in the $V_{i}$ we have to multiply $(18)$ by $(-1)^{n-1}$, as indicated before in $\S 2$. 
5.2 The number and types of singularities in $S^{k-1}$ will give us valuable information about the modes of approach to infinity of our original vector field in $E^{k}$. In this connection, it can be proved by standard methods that the set $\mathscr{H}$ of all systems $X \in \mathscr{X}$ such that the singular points of $\pi_{\infty}(X)$ are hyperbolic, and hence finite in number, is open and dense in $\mathscr{X}$.

5.3 We now restrict our attention to a very particular case, that in which $X$ is a gradient field. To illustrate the reason for this restriction we shall consider the case in which $k=3$. Here it is natural to investigate whether or not the set of all $X \in \mathscr{X}$ for which $\pi_{\infty}(X)$ is structurally stable in $S^{2}$ is open and dense. Since every perturbation in $\mathscr{X}$ is global, a natural idea to break any possible connection between saddle points is to rotate $\pi_{\infty}(X)$ in $S^{2}$. However, we can show, by giving an example, that, given an arbitrary $X \in \mathscr{X}$, it is not always possible to find a perturbation $\tilde{X} \in \mathscr{X}$ such that the corresponding $\pi_{\infty}(\tilde{X})$ is a rotation of $\pi_{\infty}(X)$.

Let our example be $X=(P, Q, R)=\left(y^{2}+z^{2}, y^{2}, z^{2}\right)$. Then, in $U_{1}, \pi_{\infty}(X)=$ $(1 / \Delta(s, t))(Q-s P, R-t P)=(1 / \Delta(s, t))\left(s^{2}-s^{3}-s t^{2}, t^{2}-t^{3}-t s^{2}\right)$. Now, for every $(s, t) \in U_{1}$, let the matrix

$$
\mathscr{R}=\left[\begin{array}{ll}
a(s, t) & b(s, t) \\
c(s, t) & d(s, t)
\end{array}\right]
$$

be such that it represents a rotation at $(s, t)$. The rotated vector is

$$
\begin{aligned}
(1 / \Delta(s, t))\left[a(s, t)\left(s^{2}-s^{3}-s t^{2}\right)+b(s, t)\left(t^{2}-t^{3}-t s^{2}\right), c(s, t)\left(s^{2}-s^{3}-s t^{2}\right)\right. & \\
& \left.+d(s, t)\left(t^{2}-t^{3}-t s^{2}\right)\right]
\end{aligned}
$$

The functions $a, b, c$, and $d$ are assumed to be continuous. If this rotated vector field comes from a system $\tilde{X} \in \mathscr{X}$, the two components inside the brackets must be polynomials of degree at most three. This implies that $\mathscr{R}$ must be a constant matrix. Moreover, the first component, $\widetilde{Q}-s \widetilde{P}$, cannot have a term in $t^{3}$, and the second, $\tilde{R}-t \tilde{P}$, cannot have a term in $s^{3}$. This implies that $b(s, t) \equiv c(s, t) \equiv 0$. Now, let $k$ be the coefficient of the term in $y^{2}$ of $\tilde{P}$. Then it is clear that it must be $a=k$ and $d=k$, that is, $a=d$. Thus, if the perturbation $\tilde{X}$ belongs to $\mathscr{X}$, then $\pi_{\infty}(\tilde{X})$ is not a rotation of $\pi_{\infty}(X)$.

However, suppose $X$ is a gradient field. Then, since the nonwandering set of $X$ is composed of a finite number of hyperbolic singularities, it is natural to expect that a similar situation holds for $\pi_{\infty}(X)$. We shall need the following definition:

Definition 3. Let $X$ be a differentiable vector field on a differentiable manifold. $X$ is said to be gradient-like if and only if:

(a) $X$ has a finite number of zeros, all of them hyperbolic, and

(b) The nonwandering set, $\Omega(X)$, coincides with the set of zeros of $X$.

This definition differs from that of Smale in that we do not require stable and unstable manifolds of hyperbolic singularities to be transversal. 
5.4 THEOREM 4. Let $f$ be a polynomial function of degree $n+1 f: E^{k} \rightarrow E^{1}$, and let $X=$ grad $f$. Then $\Omega\left(\pi_{\infty}(X)\right)$ consists of singular points only. Moreover, in the set of all gradient vector fields in $X$, there is an open and dense subset such that $\pi_{\infty}(X)$ is gradient-like $e^{(2)}$.

Proof. The second part of the theorem is proved by the same methods as in §5.2. To prove the first part we shall first find a function $g: S^{k-1} \rightarrow E^{1}$ such that $g$ is increasing along trajectories of $\pi_{\infty}(X)$, and so no closed orbits can be found in $S^{k-1}$. Also, the existence in $S^{k-1}$ of closed curves which are invariant and composed of a finite number of trajectories of $\pi_{\infty}(X)$ is clearly impossible. We shall carry out the proof for $k=3$, but the same method is applicable for arbitrary $k$.

Now, in $U_{1} \cup V_{1}$ we define $g$ by

$$
g(s, t)=f_{n+1}(1, s, t) / \Delta(s, t)^{n+1},
$$

where $f_{n+1}$ denotes the terms of the highest degree in $f$. Similar expressions hold for the other neighborhoods.

Then, let $s(\tau), t(\tau)$ be a trajectory or piece of trajectory in $U_{1} \cup V_{1}$, and let us compute the derivative of $g$ along this trajectory.

$$
d g / d \tau=(\partial g / \partial s) \dot{s}+(\partial g / \partial t) \dot{t}
$$

But $\dot{s}$ and $i$ are given by the two components of the first expression in (18). Therefore

$$
\begin{aligned}
\frac{d g}{d \tau}= & \frac{\Delta^{n+1}\left(\partial f_{n+1} / \partial s\right)-(n+1) f_{n+1} s \Delta^{n-1}}{\Delta^{2 n+2}} \frac{Q_{n}-s P_{n}}{\Delta^{n-1}} \\
& +\frac{\Delta^{n+1}\left(\partial f_{n+1} / \partial t\right)-(n+1) f_{n+1} t \Delta^{n-1}}{\Delta^{2 n+2}} \frac{R_{n}-t P_{n}}{\Delta^{n-1}} \\
= & \left(1 / \Delta^{2 n+2}\right)\left[\left(\Delta^{2} Q_{n}-(n+1) f_{n+1} s\right)\left(Q_{n}-s P_{n}\right)\right. \\
& \left.+\left(\Delta^{2} R_{n}-(n+1) f_{n+1} t\right)\left(R_{n}-t P_{n}\right)\right] .
\end{aligned}
$$

$\left({ }^{2}\right)$ After this paper had been submitted for publication, Professor Jacob Palis suggested that Theorem 4 can be improved by substituting its second half by the statement: "Moreover, in the set of all gradient vector fields in $X$, there is an open and dense subset such that $\pi_{\infty}(X)$ is S-stable."

The proof of this fact follows from the following modification of a result of Palis: "If $V$ is a gradient-like field and has the no cycle property on $\Omega(V)$, then $V$ is $\Omega$-stable." [See J. Palis, On Morse-Smale dynamical systems, to appear in Topology.]

We recall that if $V$ is gradient-like and $\Omega(V)=\left\{\alpha_{i_{1}}, \ldots, \alpha_{i_{n}}\right\}$ is its set of zeros, then $V$ has the no cycle property if there is no sequence $\alpha_{i_{1}}, \ldots, \alpha_{i_{k}}$ in $\Omega(V)$ such that $W^{s}\left(\alpha_{i_{j}}\right) \cap W^{u}\left(\alpha_{i_{j}+1}\right)$ $\neq \varnothing$ for all $1 \leqq j \leqq k-1, i_{1}=i_{k}$, and $i_{1} \neq i$, for some $j$. Here $W^{s}$ and $W^{u}$ stand for the stable and unstable manifolds respectively. For the definition of $\Omega$-stability see [9].

In our case, the fact that $g$ is increasing along trajectories implies that $\pi_{\infty}(X)$ has the no cycle property.

I want to thank Professor Palis for communicating this note to me. 
Since $f$ is homogeneous of degree $n+1$ we know that $(n+1) f_{n+1}=x\left(\partial f_{n+1} / \partial x\right)$ $+y\left(\partial f_{n+1} / \partial y\right)+z\left(\partial f_{n+1} / \partial z\right)$. Then, in terms of $s$ and $t$, we have $(n+1) f_{n+1}=P_{n}$ $+s Q_{n}+t R_{n}$, and inserting this into (21) we get

$$
\begin{aligned}
\frac{d g}{d \tau}= & \frac{1}{\Delta^{2 n+2}}\left[\left(\left(1+t^{2}\right) Q_{n}-s P_{n}-s t R_{n}\right)\left(Q_{n}-s P_{n}\right)\right. \\
& \left.+\left(\left(1+s^{2}\right) R_{n}-t P_{n}-s t Q_{n}\right)\left(R_{n}-t P_{n}\right)\right] \\
= & \frac{1}{\Delta^{2 n+2}}\left[\left(Q_{n}-s P_{n}\right)^{2}+\left(R_{n}-t P_{n}\right)^{2}+\left(t Q_{n}-s R_{n}\right)^{2}\right]
\end{aligned}
$$

We observe that this expression vanishes at the zeros of $\pi_{\infty}(X)$ and that these are the only points at which it vanishes. Anywhere else it is positive.

The process is valid in the other neighborhoods. Thus, we conclude that $d g / d \tau>0$ along every trajectory which is not a zero of $\pi_{\infty}(X)$.

To complete the proof, let $p=\left(s_{0}, t_{0}\right)$ be a nonwandering point which is not a zero of $\pi_{\infty}(X)$, and let $\phi_{\tau}$ be the flow associated with this field. Then, for every neighborhood, $N$, of $p$ there exists a sequence $\left\{\tau_{i}\right\} \rightarrow \infty$ such that $\left(\phi_{\tau_{i}}(N)\right) \cap N \neq \varnothing$. Now let $K=\sup _{\tau}\left[g\left(\phi_{\tau}(p)\right)-g(p)\right]$. By the continuity of $g$ we can choose the neighborhood $N$ so small that $\sup _{q \in N}[g(q)-g(p)]<K$, a contradiction. This implies that every nonwandering point of $\pi_{\infty}(X)$ must be a singular point. The proof of the theorem is thus complete.

6. Classification of the systems in $\mathscr{X}$ for $\dot{x}=A x$ and $k=3$.

6.1 For fields of the form $X(x)=A x$, with $A$ a constant matrix, the coefficient space is $\mathscr{X}=E^{9}$. The zeros of this field are located at infinity and at the origin. At the origin the equation is linear and can be easily solved. Regarding the behavior at infinity, we shall give a decomposition of $\mathscr{X}$ into eleven classes of equivalence, such that $X$ and $Y$ belong to the same class if and only if the flows corresponding to $\pi_{\infty}(X)$ and $\pi_{\infty}(Y)$ are topologically equivalent. Each class of equivalence can be shown to be an algebraic set in $\mathscr{X}$.

A further study of the possible types of the singularity at the origin is also included. Then, it is seen that we can have a nonhyperbolic singularity at the origin while everything is hyperbolic at infinity, and vice versa.

6.2 For any two systems $X$, and $Y$, in $\mathscr{X}$, for which the real Jordan canonical form of $A$ is the same, $\pi_{\infty}(X)$ and $\pi_{\infty}(Y)$ are topologically equivalent.

There are four types of real Jordan form:

$$
\begin{array}{ccc}
\text { I }\left[\begin{array}{lll}
\lambda & & \\
& \mu & \\
& & \nu
\end{array}\right], & \operatorname{II}\left[\begin{array}{lll}
\lambda & 1 & \\
& \lambda & \\
& & \mu
\end{array}\right], \\
\operatorname{III}\left[\begin{array}{lll}
\lambda & 1 & \\
& \lambda & 1 \\
& & \lambda
\end{array}\right], & \operatorname{IV}\left[\begin{array}{ccc}
\alpha & -\beta & \\
\beta & \alpha & \\
& & \lambda
\end{array}\right] .
\end{array}
$$


In Case $\mathrm{I}, \pi_{\infty}(X)$ is given by:

$$
[(\mu-\lambda) s,(\nu-\lambda) t], \quad[(\lambda-\mu) s,(\nu-\mu) t], \quad \text { and }[(\lambda-\nu) s,(\mu-\nu) t],
$$

in $U_{1}, U_{2}$, and $U_{3}$ respectively, and the same for the $V$ 's. We observe that the zeros of $\pi_{\infty}(X)$ will be isolated if and only if the eigenvalues are distinct. If this is the case, we shall have two contracting nodes, two expanding nodes and two saddle points at infinity. If we denote by $\mathscr{G}$ the set of all systems $X$ in $\mathscr{X}$ such that $\pi_{\infty}(X)$ is a Morse-Smale system, then, in Case $\mathrm{I}, X \in \mathscr{G}$ if and only if the eigenvalues of the Jacobian matrix are distinct.

In Case II, $\pi_{\infty}(X)$ is given by:

$$
\left[-s^{2},(\mu-\lambda) t-s t\right], \quad[1,(\mu-\lambda) t], \quad \text { and } \quad[(\lambda-\mu) s+t,(\lambda-\mu) t] .
$$

The point $(0,0) \in U_{1}$ is always a nonhyperbolic singularity. Then, no $X$ is in $\mathscr{G}$ in this case.

The same thing happens in Case III, since $\pi_{\infty}(X)$ is given by:

$$
\left(t-s^{2},-s t\right), \quad\left(1-s t,-t^{2}\right), \quad \text { and }(t, 1) \text {. }
$$

The only zeros are $(0,0) \in U_{1}$ and $(0,0) \in V_{1}$, and they are never hyperbolic. Notice that $\pi_{\infty}(X)$ does not depend on $\lambda$.

In Case IV, $\pi_{\infty}(X)$ is given by:

$$
\begin{gathered}
{\left[\beta\left(1+s^{2}\right),(\lambda-\alpha+\beta s) t\right], \quad\left[-\beta\left(1+s^{2}\right),(\lambda-\alpha-\beta s) t\right], \quad \text { and }} \\
{[(\alpha-\lambda) s-\beta t,(\alpha-\lambda) t+\beta s] .}
\end{gathered}
$$

The only zeros are $(0,0) \in U_{3}$ and $(0,0) \in V_{3}$. In the other neighborhoods, the set $t=0$ is invariant. It represents a closed orbit, $C$, with stability index given by:

$$
\begin{aligned}
h(C)= & 2 \int_{U_{1}(1)}(2 \beta s+\lambda-\alpha+\beta s)_{s=s(\tau) ; t=0} d \tau \\
& +2 \int_{U_{2}(1)}(-2 \beta s+\lambda-\alpha-\beta s)_{s=s(\tau) ; t=0} d \tau \\
= & 4 \int_{U_{1}(1)}(\lambda-\alpha) d \tau .
\end{aligned}
$$

The behavior of $\pi_{\infty}(X)$ depends only on $\beta$ and the difference $\lambda-\alpha . \beta$ is always different from zero, and can be considered to be positive. Otherwise, make the change $x_{1} \rightarrow x_{2}, x_{2} \rightarrow x_{1}, x_{3} \rightarrow x_{3}$. $C$ is hyperbolic if and only if $\lambda-\alpha \neq 0$. This is also the condition for the two zeros to be each a focus instead of a center. $C$ is contracting when $\lambda-\alpha<0$, and then the two foci are expanding; $C$ is expanding and the two foci are contracting when $\lambda-\alpha>0$. For $\lambda-\alpha>0$ the same picture, but upside down and with the direction of the arrows reversed.

6.2 We have seen that there are three possible types of qualitative behavior at infinity for systems in $\mathscr{G}$ :

$\mathrm{G} 1: A$ has real distinct eigenvalues. 
G2: $A$ has one real eigenvalue, $\lambda$, and two complex conjugate eigenvalues, $\alpha \pm \beta i$, with $\lambda-\alpha>0$.

G3: The same as in G2, but with $\lambda-\alpha<0$.

Any two systems in the same $\mathrm{G} i$ are topologically conjugate at infinity. The construction of the homeomorphism is done as in [6].

Let $\lambda^{3}+a \lambda^{2}+b \lambda+c=0$ be the characteristic equation of $A$. We know that $a=-\operatorname{tr} A$ and $c=-\operatorname{det} A$. Also, from Cardano's solution to the third order equation, we know that $X$ is in G1 if and only if $D=-3\left(a^{2} b^{2}-4 a^{3} c-4 b^{3}+18 a b c\right.$ $\left.-27 c^{2}\right)<0 . X$ is in G2 if and only if $D>0$ and $h=-c / 2+a b / 6-a^{3} / 27>0$, and $X$ is in G3 if and only if $D>0$ and $h<0$.

Thus, we have completely described the algebraic sets in $\mathscr{X}$ which correspond to systems in $\mathscr{G}$.

6.3 We can do the same thing for systems in $\mathscr{X}-\mathscr{G}$. There are eight possible types of qualitative behavior at infinity:

NG1: $D>0$ and $h=0$. Then $\lambda=\alpha$ and, from (27), we get that $(0,0) \in U_{3}$ and $(0,0) \in V_{3}$ are centers, and $C$ belongs to a ring of closed orbits.

The rest of the cases correspond to $D=0$. If we also have $h>0$, then two and only two of the eigenvalues of $A$ are equal. There are two cases, which depend on whether the Jordan form of $A$ is of the type I, with $\lambda=\mu$, or of the type II. We have, respectively:

NG2: From (24), we know that $(0,0) \in U_{3}$ and $(0,0) \in V_{3}$ are the only zeros of $\pi_{\infty}(X)$. Since, in this case, $h=2(\nu-\lambda)^{3} / 27$ we get that $\nu>\lambda=\mu$. This means that the two zeros are contracting nodes. In the other neighborhoods, all the points of the form $(s, 0)$ represent a circle, which is composed of zeros of $\pi_{\infty}(X)$.

NG3: $\pi_{\infty}(X)$ is given by (25) with $\mu>\lambda$. The zeros are $(0,0) \in U_{1},(0,0) \in V_{1}$, $(0,0) \in U_{3}$, and $(0,0) \in V_{3}$. The first two are never hyperbolic, as is easily seen after computing the Jacobian matrix. The others are degenerate nodes. In this, and all the other cases as well, it is a simple matter to construct the corresponding picture for $\pi_{\infty}(X)$.

When $D=0$ and $h<0$, the situation is entirely similar:

NG4: As in NG2, but the nodes are expanding.

NG5: $\pi_{\infty}(X)$ is topologically as in NG3, but with the direction of the arrows reversed.

It remains to study the behavior of $\pi_{\infty}(X)$ when both $D$ and $h$ are zero. There are three possibilities, depending on whether the Jordan form of $A$ is of the type I, II, or III, respectively:

NG6: $\pi_{\infty}(X) \equiv 0$.

NG7: There is a circle, $C$, composed of zeros of $\pi_{\infty}(X)$, and all the other trajectories approach $C$ as $t \rightarrow \pm \infty$.

NG8: $\pi_{\infty}(X)$ is given by (26), which is independent of $\lambda$. Thus, $\pi_{\infty}(X)$ is unique for all systems of this type. The only zeros, which are never hyperbolic, are $(0,0) \in U_{1}$ and $(0,0) \in V_{1}$. 
Again, in each one of these eight cases, the construction of the homeomorphism is done as in [6], although new types of canonical regions arise.

Only the algebraic set corresponding to NG1 has been characterized. Further distinctions have to be made in the other three groups. Since the characteristic equation is the same within each of these groups, we shall now consider the minimal polynomial, $m(x)$. For NG2 and NG4, it is given by $(x-\lambda)(x-\nu)$; for NG3 and NG5, by $(x-\lambda)^{2}(x-\mu)$; for NG6, by $x-\lambda$; for NG7, by $(x-\lambda)^{2}$; and for NG8, by $(x-\lambda)^{3}$. For NG6, the corresponding class of equivalence in $\mathscr{X}$ is given by the intersection of the algebraic variety $D=0, h=0$ with the one which corresponds to all $3 \times 3$ matrices which are multiples of the identity. For NG2, we shall have the intersection of $D=0, h>0$ with the union of three algebraic varieties, corresponding to all $3 \times 3$ matrices for which $m(x)$ is of the second degree. For example, if $A=\left[a_{i j}\right]$, one of these three varieties is: $a_{22}=a_{33}, a_{12} a_{23}=0, a_{31} a_{23}=0, a_{13} a_{21}=0$, $a_{21} a_{32}=0, a_{11} a_{23}-a_{13} a_{21}=a_{23} a_{33}$, and $a_{11} a_{23}-a_{12} a_{31}=a_{32} a_{33}$. This is obtained from the condition that the elements of the adjoint of the characteristic matrix of $A$ have one common factor. It is easy to see that there are two other varieties for which this is so, and the first equations of each one of these are: $a_{11}=a_{22}$, and $a_{11}=a_{33}$, respectively.

We can do the same for the other NG, thus completing the study of all classes of equivalence in $\mathscr{X}$ for which we have a different qualitative behavior of $\pi_{\infty}(X)$.

6.4 For systems of the form $X(x)=A x$, there is only one more zero of the Poincaré field, and it is located at the origin of $E^{3}$. For any one of the eleven cases considered in 6.2 and 6.3 , there will be several possibilities at the origin. For instance, when $D<0, X$ is in $\mathrm{G} 1$ and $A$ has distinct eigenvalues. Then, there are seven subcases (the notation is selfexplanatory; the small $g$ in some of the subcases indicates that the origin is hyperbolic):

G1. 1g: The eigenvalues are positive. Then the origin, 0 , is an expanding node of $\pi(X)$. If we recall the expression of the characteristic equation, this case corresponds to the set $D<0, c<0, a<0$, and $a b-c=-[(\mu+\nu) \mu \lambda-a \lambda(\mu+\nu)]<0$. We observe that the last two conditions are the equivalent to the Routh-Hurwitz conditions for a third order equation to have roots with positive real parts.

G1. $2 \mathrm{~g}$ : The eigenvalues are negative. Then 0 is a contracting node of $\pi(X)$. The corresponding set is $D<0, c>0, a>0$, and $a b-c>0$.

Gl. $3 \mathrm{~g}$ : One eigenvalue is positive and two negative. 0 is hyperbolic and the stable manifold is 2 -dimensional. The set is given by $D<0, c<0$, and either $a \geqq 0$ or else $a<0$ and $a b-c>0$.

G1. $4 \mathrm{~g}$ : One eigenvalue is negative and two positive. 0 is hyperbolic and the stable manifold is 1-dimensional. The set is given by $D<0, c>0$, and either $a \leqq 0$ or else $a>0$ and $a b-c<0$.

G1. 5: One eigenvalue is zero and two positive. 0 is not hyperbolic, but has a 2-dimensional unstable manifold (these manifolds are always planes or lines). The set is $D<0, c=0, a<0$, and $b>0$. 
G1. 6: One eigenvalue is zero and two negative. 0 is not hyperbolic, but has a 2-dimensional stable manifold. The set is $D<0, c=0, a>0$, and $b>0$.

G1. 7: One eigenvalue is zero and the other two of opposite sign. 0 is not hyperbolic, but has 1-dimensional stable and unstable manifolds. The set is $D<0$, $c=0$, and $b<0$.

We observe that all the sets are mutually exclusive, and their union is the set $D<0$. We also notice that the origin can be hyperbolic or not, while the behavior of $\pi_{\infty}(X)$ is always hyperbolic. We shall see that the opposite situation can actually happen.

Let us now deal with the rest of the systems in $\mathscr{G}$. They are in $D>0, h \neq 0$. We have:

G2. 1g: $\lambda>\alpha>0.0$ is expanding, and a focus in a 2-manifold. The set is $D>0$, $h>0, c<0, a<0$, and $a b-c=-2\left[\alpha^{3}+\alpha \beta^{2}-a \alpha \lambda\right]<0$.

G2. 2: $\lambda>\alpha=0.0$ is not hyperbolic, it is a center in a 2-manifold, and otherwise expanding. The set is $D>0, h>0, c<0, a<0$, and $a b-c=0$.

G2. $3 g: \lambda>0>\alpha .0$ is a contracting focus in a 2-manifold, and otherwise expanding. The set is $D>0, h>0, c<0$, and either $a \geqq 0$ or else $a<0$ and $a b-c>0$.

G2. 4: $0=\lambda>\alpha .0$ is not hyperbolic, but is a contracting focus in a 2-manifold. The set is $D>0, h>0, c=0$.

G2. $5 \mathrm{~g}: 0>\lambda>\alpha .0$ is contracting, and a focus in a 2 -manifold. The set is $D>0, h>0, c>0$.

G3. 1g: $\alpha>\lambda>0.0$ as in G2. 1g. The set is $D>0, h<0, c<0$.

G3. 2: $\alpha>\lambda=0.0$ as in G2. 4 but with the arrows reversed. The set is $D>0$, $h<0, c=0$.

G3. $3 \mathrm{~g}: \alpha>0>\lambda .0$ as in G2. $3 \mathrm{~g}$ but with the arrows reversed. The set is $D>0$, $h<0, c>0$, and either $a \leqq 0$ or else $a>0$ and $a b-c<0$.

G3. 4: $0=\alpha>\lambda$. 0 as in G2. 2 but with the arrows reversed. The set is $D>0$, $h<0, c>0, a>0$, and $a b-c=0$.

G3. 5g: $0>\alpha>\lambda .0$ as in G1. 5g. The set is $D>0, h<0, c>0, a>0$, and $a b-c>0$. Again, we have found cases in which $\pi_{\infty}(X)$ is hyperbolic but the origin is not. There are no more systems in $\mathscr{G}$. The other cases are as follows:

NG1. 1g: $\lambda=\alpha>0$. 0 as in G2. 1g. The set is $D>0, h=0, c<0$.

NG1. 2: $\lambda=\alpha=0.0$ is a center in a 2-manifold and the other 1-manifold is singular. The set is $D>0, h=0, c=0$.

NG1. 3g: $0>\lambda=\alpha .0$ as in G2. 5g. The set is $D>0, h=0, c>0$.

The rest of the systems are in the algebraic variety $D=0$. Since we already know how to distinguish the different sets as far as the sign of $h$ and the degree of the minimal polynomial, we shall now indicate only the rest of the distinguishing features. We have:

NG2. $1 \mathrm{~g}: \nu>\lambda>0$, where $\lambda$ is the repeated eigenvalue. 0 is an expanding node, and the set is $c<0, a<0$, and $a b-c=-2\left(\lambda^{3}-a \lambda \nu\right)<0$.

NG2. $2 \mathrm{~g}: \lambda<\nu<0.0$ is a contracting node, and the set is $c>0$. 
NG2. 3g: $\lambda<0<\nu .0$ is contracting in a 2-manifold and expanding in a 1-manifold. The set is $c<0$, and either $a \geqq 0$ or else $a<0$ and $a b-c>0$.

NG2. 4: $\nu>\lambda=0.0$ is expanding in a 1-manifold, and has a singular 2-manifold. The set is $c=0, a<0$.

NG2. 5: $\lambda<\nu=0.0$ is contracting in a 2-manifold, and has a singular 1-manifold. The set is $c=0, a>0$.

NG3. 1g: $\mu>\lambda>0.0$ is an expanding node, degenerate in a 2-manifold. The set is $c<0, a<0$, and $a b-c=-2\left(\lambda^{3}-a \lambda \mu\right)<0$.

NG3. 2: $\mu>\lambda=0$. The degenerate node in NG3. $1 \mathrm{~g}$ is transformed in a singular line, and the field is parallel to this line with opposite directions at its two sides. The set is $c=0, a<0$.

NG3. $3 g: \mu>0>\lambda .0$ is a degenerate contracting node in a 2-manifold, and otherwise expanding. The set is $c<0$, and either $a \geqq 0$ or else $a<0$ and $a b-c>0$.

NG3. 4: $\mu=0>\lambda$. 0 is a degenerate contracting node in a 2-manifold, and has a singular 1-manifold. The set is $c=0, a>0$.

NG3. $5 \mathrm{~g}: 0>\mu>\lambda .0$ is a contracting node, degenerate in a 2 -manifold. The set is $c>0$.

NG4. 1g: $\lambda>\nu>0.0$ as in NG2. $1 \mathrm{~g}$, and the set is $c<0$.

NG4. $2 \mathrm{~g}: \nu<\lambda<0.0$ as in NG2. $2 \mathrm{~g}$, and the set is $c>0, a>0$, and $a b-c>0$.

NG4. $3 \mathrm{~g}: \nu<0<\lambda .0$ as in NG2. $3 \mathrm{~g}$ but with the arrows reversed. The set is $c>0$, and either $a \leqq 0$ or else $a>0$ and $a b-c<0$.

NG4. 4: $\nu<\lambda=0.0$ as in NG2.4 but with the arrows reversed. The set is $c=0$, $a>0$.

NG4. 5: $\lambda>\nu=0.0$ as in NG2. 5 but with the arrows reversed. The set is $c=0$, $a<0$.

NG5. 1g: $\lambda>\mu>0.0$ as in NG3. 1g. The set is $c<0$.

NG5. $2 \mathrm{~g}: \lambda>\mu=0.0$ as in NG3. 4 but with the arrows reversed. The set is $c=0$, $a<0$.

NG5. $3 \mathrm{~g}: \lambda>0>\mu .0$ as in NG3. 3g. The set is $c>0$, and either $a \leqq 0$ or else $a>0$ and $a b-c<0$.

NG5. 4: $0=\lambda>\mu .0$ as in NG3. 2 but with the arrows reversed. The set is $c=0$, $a>0$.

NG5. 5g: $0>\lambda>\mu .0$ as in NG3. 5g. The set is $c>0, a>0$, and $a b-c>0$.

NG6. $1 \mathrm{~g}: \lambda>0.0$ is an expanding node, and the set is $c<0$.

NG6. $2 \mathrm{~g}: \lambda=0 . \pi(X) \equiv 0$, and the set is $c=0$.

NG6. $3 \mathrm{~g}: \lambda<0.0$ is a contracting node, and the set is $c>0$.

NG7. 1g: $\lambda>0.0$ is an expanding node, degenerate in a 2-manifold. The set is $c<0$.

NG7. 2: $\lambda=0$. Two axes are composed of zeros. In a plane containing one of these axes the field is parallel to it, with opposite directions at both sides. The set is $c=0$.

NG7. $3 \mathrm{~g}: \lambda<0.0$ is a contracting node, degenerate in a 2 -manifold. The set is $c>0$. 
NG8. 1g: $\lambda>0.0$ is an expanding node. The set is $c<0$.

NG8. 2: $\lambda=0.0$ is nonhyperbolic. One axis is composed of zeros, and there is a plane in which $\pi(X)$ is parallel to this axis, with opposite directions at both sides. The set is $c=0$.

NG8. $3 \mathrm{~g}: \lambda<0.0$ is a contracting node. The set is $c>0$.

We see that, as remarked before, there are systems for which the origin is hyperbolic, but not so all the elements of $\Omega\left(\pi_{\infty}(X)\right)$, and vice versa. It is easy to check that if $X \in \mathscr{G}$ and also the origin is hyperbolic, then $\Omega(\pi(X))$ is composed of only hyperbolic elements. The converse is also true.

\section{BIBLIOGRAPHY}

1. H. Poincaré, Mémoire sur les courbes définies par une equation différentielle, J. Mathématiques (3) 7 (1881), 375-422.

2. É. Picard, Traité d'analyse, Tome III, Gauthier-Villars, Paris, 1896, pp. 228-232.

3. G. Sansone and R. Conti, Equazioni differentiali non lineari, Edizione Cremonese, Rome, 1956, pp. 122-133.

4. T. V. Davies and E. M. James, Nonlinear differential equations, Addison-Wesley, Reading, Mass., 1966, pp. 42-46.

5. M. M. Peixoto, On structural stability, Ann. of Math. (2) 69 (1959), 199-222.

6. M. C. Peixoto and M. M. Peixoto, Structural stability in the plane with enlarged boundary conditions, An. Acad. Brasil. Ci. 31 (1959), 135-160.

7. I. Kupka, Contribution à la théorie des champs génériques, Contributions to Differential Equations 2 (1963), 457-484.

8. S. Smale, On dynamical systems, Bol. Soc. Mat. Mexicana 5 (1960), 195-198.

9. —_ Differentiable dynamical systems, Bull. Amer. Math. Soc. 73 (1967), 747-816.

10. S. Lefschetz, Differential equations: geometric theory, Interscience, New York, 1963, pp. 201-208.

11. H. Weber, Lehrbuch der Algebra, Friedrich Vieweg und Sohn, Braunschweig, 1895, pp. $152,157$.

Boston College,

Chestnut Hill, Massachusetts 